\title{
Expression and significance of miR-24 and miR-101 in patients with advanced gastric cancer
}

\author{
XUETAO DONG and YANDI LIU \\ Department of Gastroenterology, Tianjin Union Medical Centre, Tianjin 300120, P.R. China \\ Received March 5, 2018; Accepted August 7, 2018
}

DOI: $10.3892 / 01.2018 .9324$

\begin{abstract}
Clinical significance of micro ribonucleic acid (miR)-24 and miR-101 were investigated by evaluating the expression of miR-24 and miR-101 in the tissues of patients with advanced gastric cancer. A total of 247 gastric cancer tissue specimens and 150 cancer-adjacent normal tissues ( $>5 \mathrm{~cm}$ away from the tumor) from patients with advanced gastric cancer who underwent surgical resection in the Surgical Oncology Department of Tianjin Union Medical Centre (Tianjin, China) from April 2013 to May 2016 were collected. The expression of miR-24 and miR-101 in gastric cancer and cancer-adjacent normal tissues were detected via reverse transcription-quantitative polymerase chain reaction (RT-qPCR), and the correlation of the levels of miR-24 and miR-101 in gastric cancer tissues with their clinical and pathological features were explored. The expression level of miR-24 in gastric cancer tissues was significantly higher than that in cancer-adjacent normal tissues $(\mathrm{t}=10.26, \mathrm{p}<0.01)$, while the expression level of miR-101 was significantly lower $(\mathrm{t}=13.940, \mathrm{p}<0.01)$. The expression of $\mathrm{miR}-24$ and $\mathrm{miR}-101 \mathrm{in}$ gastric cancer was correlated with the pathological differentiation degree of the tumor, lymph node metastasis and depth of infiltration $(\mathrm{p}<0.05)$. The multivariate Cox regression analysis revealed that miR-24 and miR-101 were independent prognostic factors affecting the overall survival of patients $(\mathrm{p}<0.01)$. The results indicated that the expression of miR-24 is upregulated and that of miR-101 is downregulated in gastric cancer tissues. miR-24 and miR-101 may promote the occurrence, development, infiltration and metastasis of gastric cancer, and can be indicators for the prognosis of patients with gastric cancer.
\end{abstract}

Correspondence to: Dr Yandi Liu, Department of Gastroenterology, Tianjin Union Medical Centre, 190 Jieyuan St. W., Hongqiao District, Tianjin 300120, P.R. China

E-mail: liuyd66n@163.com

Key words: micro ribonucleic acid-24, micro ribonucleic acid-101, advanced gastric cancer, degree of pathological differentiation, lymph node metastasis, infiltration

\section{Introduction}

Gastric cancer is the most common gastrointestinal cancer in human and originates from malignant tumors of epithelial cells. High-prevalence groups of gastric cancer are the population aged 55-75 years, and its incidence rate in men (ranks fourth among cancers worldwide) is slightly higher than that in women (ranks fifth among cancers worldwide) (1). In recent years, with the development of the society, patients with gastric cancer tend to be increasingly younger, which is closely related to the living environment and habits. According to data provided by the World Health Organization (WHO), there are $>1.5$ million patients with gastric cancer around the world, and the mortality rate caused by gastric cancer ranks second among the malignant tumors (2). At present, the treatment for patients with gastric cancer and their survival time are not optimistic, and the survival time of patients with early gastric cancer is greatly prolonged compared with that of patients with advanced gastric cancer. The treatment for gastric cancer is mainly based on radical surgery, supplemented by chemotherapy, radiotherapy and other non-surgical treatments (3). Exacerbated gastric cancer affects the survival of patients, and during the exacerbation, tumor cell invasion and metastasis will gradually appear. The metastasis of tumor cells is a multi-factor involved and complex process, during which the cells metastasize from the primary focus to the tissue nearby. After metastasis, tumor cells can invade the vasculature, and proliferate to form secondary tumors, thus leading to the re-development of gastric cancer and resulting in death (4).

The formation and development of gastric cancer are very complicated processes, and no ideal biological prediction indicator for the occurrence, development, invasion, metastasis and prognosis of gastric cancer has been found so far. According to relevant reports, some micro ribonucleic acids (miRNAs) are expressed in gastric cancer and involved in the pathological and physiological development processes of gastric cancer (5). miRNA regulates the post-transcriptional gene level, plays important roles in a series of activities such as growth, senescence and death of organisms, and plays a carcinogenic or tumor suppressive role in tumors (6). Therefore, the role of miRNA in the tumor has been the focus of clinical research, and miRNA plays key roles in the processes of tumorigenesis, development and treatment. miR-24 is widely distributed in various tissues and organs of the human body, participates in 
multi-tissue and systemic cell differentiation of the body and plays an important role in the process of differentiation (7). miR-101, as a tumor suppressor gene, has two precursors, located in human chromosomes 1 and 9, and its mature sequence is highly conserved in various species (8).

At present, there is little research on the expression of miR-24 and miR-101 in gastric cancer, and the combination of both for monitoring the status of gastric cancer has not been reported yet. This study investigated the roles of miR-24 and miR-101 in gastric cancer by observing their expression so as to provide evidence for the treatment of gastric cancer.

\section{Materials and methods}

General data. A total of 247 gastric cancer tissue specimens and 150 cancer-adjacent normal tissues ( $>5 \mathrm{~cm}$ away from the tumor) were collected from patients with advanced gastric cancer who underwent surgical resection in the Surgical Oncology Department of Tianjin Union Medical Centre from April 2013 to May 2016. The patients were aged 30-75 years, with an average age of $45.36 \pm 7.26$ years, including 135 males and 112 females. According to the WHO clinicopathological classification and staging criteria for gastric tumors (2010) (9), patients were divided into high differentiation $(n=34)$, moderate differentiation $(n=60)$ and low differentiation $(n=153)$ in light of the tumor differentiation degree, into stage III $(n=114)$ and stage IV $(n=133)$ on the basis of their clinical stages, and into $\mathrm{N} 0+\mathrm{N} 1(\mathrm{n}=131)$ and $\mathrm{N} 2+\mathrm{N} 3(\mathrm{n}=116)$ based on the state of lymph node metastasis. Inclusion criteria: patients whose cancer tissues were confirmed by the gold standard of the Pathology Department, those whose cancer tissues were immediately placed in liquid nitrogen after surgical resection and stored in a refrigerator $\left(-80^{\circ} \mathrm{C}\right)$ for low temperature storage, and those whose clinical data were complete. Exclusion criteria: patients having undergone chemoradiotherapy before surgery, those with family history of mental illness or psychosis, and those with liver metastasis or lung cancer. This study was approved by the Ethics Committee of Tianjin Union Medicine Centre (Tianjin, China). The study subjects were informed, and signed the informed consent.

Main instruments and reagents. LightCycler real-time quantitative polymerase chain reaction (PCR) instrument was purchased from Roche Diagnostics (Basel, Switzerland), the total RNA extraction kit (TRIzol method) from Invitrogen (Invitrogen: Thermo Fisher Scientific, Carlsbad, CA, USA), Moloney Murine Leukemia Virus (M-MLV) reverse transcription (RT) kit from Vazyme (Nanjing, China), and miR-24 and miR-101 fluorescence quantitative PCR kits from Biomiga (San Diego, CA, USA). miR-24, miR-101 and U6 reference primers for real-time quantitative PCR were synthesized by the Sangon Biotech (Shanghai) Co., Ltd. (Shanghai, China). The required primer sequences are shown in Table I.

Detection methods. Gastric tissue specimens preserved at $-80^{\circ} \mathrm{C}$ were taken out, and $200 \mathrm{mg}$ was cut and placed into liquid nitrogen, and then rapidly and fully grinded for standby application. TRIzol reagent was used for spare tissues, which were vibrated and let stand for $30 \mathrm{~min}$ at room temperature for full lysis. The total RNA was extracted in strict accordance with the manufacturer's instructions. The concentration and purity of the extracted RNA was determined via an ultraviolet spectrophotometer (Bio-Rad, Hercules, CA, USA) and protein electrophoresis. The extracted total RNA was reverse transcribed according to the instructions of the RT kit. The extracted complementary deoxyribonucleic acid (cDNA) specimens were stored at $-20^{\circ} \mathrm{C}$. RT-q PCR reaction system was prepared according to the instructions, which was made up to $20 \mu \mathrm{l}$ using diethyl pyrocarbonate (DEPC). PCR reaction conditions: pre-denaturation at $94^{\circ} \mathrm{C}$ for $10 \mathrm{~min}, 94^{\circ} \mathrm{C}$ for $45 \mathrm{sec}, 60^{\circ} \mathrm{C}$ for $45 \mathrm{sec}, 72^{\circ} \mathrm{C}$ for $45 \mathrm{sec}$, and a total of 40 cycles. The amplified data were analyzed using the manufacturer's software with U6 as an internal reference gene. The experiment was repeated 3 times to take an average value, and the value was considered as the amplification result, which was processed using $2^{-\Delta \Delta C q}(10)$.

Statistical analysis. Statistical analysis was performed using Statistical Product and Service Solutions (SPSS) v.19.0 (SPSS, Inc., Shanghai, China). Measurement data were expressed as mean \pm standard deviation (SD), and t-test was used for comparison of measurement data. Comparison of the mean among multiple groups were analyzed via the analysis of variance, and the post hoc test was Dunnett's test. Cox regression equation was used to analyze the independent prognosis. $\mathrm{P}<0.05$ represents that the difference was statistically significant.

\section{Results}

Expression of miR-24 in gastric cancer and cancer-adjacent normal tissues. The expression level of miR-24 was 1.906 \pm 1.231 in gastric cancer tissues and $0.836 \pm 0.437$ in cancer-adjacent normal tissues, and the expression level of miR-24 in gastric cancer tissues was significantly higher than that in cancer-adjacent normal tissues $(\mathrm{t}=10.26, \mathrm{p}<0.01)$ (Fig. 1$)$.

Expression of miR-101 in gastric cancer and cancer-adjacent normal tissues. The expression level of miR-101 was $0.316 \pm 0.229$ in gastric cancer tissues and $1.167 \pm 0.914$ in cancer-adjacent normal tissues, and the expression level of miR-101 in gastric cancer tissues was significantly lower than that in cancer-adjacent normal tissues $(t=13.940$, $\mathrm{p}<0.01$ ) (Fig. 2).

Correlation of the expression of $m i R-24$ and miR-101 in gastric cancer tissues with clinicopathological features. The expression of miR-24 and miR-101 in gastric cancer tissues was correlated with the degree of tumor differentiation, lymph node metastasis and depth of infiltration, and the differences were of statistical significance $(\mathrm{p}<0.05)$, but they were not associated with age, sex, tumor tissue type, tumor-node-metastasis (TNM) stage, tumor site and tumor size, and the differences were not statistically significant ( $>0.05)$ (Table II).

Survival curves of advanced gastric cancer patients with expression of high and low miR-24 and miR-101. According to the follow-up data, 224 out of 247 patients with advanced gastric cancer died of disease progression. To determine 
Table I. miR-24, miR-101 and U6 gene primer sequences.

\begin{tabular}{lll}
\hline Genes & Forward primer sequences & \multicolumn{1}{c}{ Reverse primer sequences } \\
\hline$m i R$ - 24 & 5'-GCTCTGGCTCAGTTCAGCAG-3' & 5'-CAGTGCAGGGTCCGAGGT-3' \\
$m i R$ - 101 & 5'-TGGGCTACAGTACTGTGATA-3' & 5'-TGCGTGTCGTGGAGTC-3' \\
U6 & 5'-CATTGCACTTGTCTCGGTCT-3' & 5'-GGTCCGAGGTATTCGCACT-3'
\end{tabular}

miR, micro ribonucleic acid.

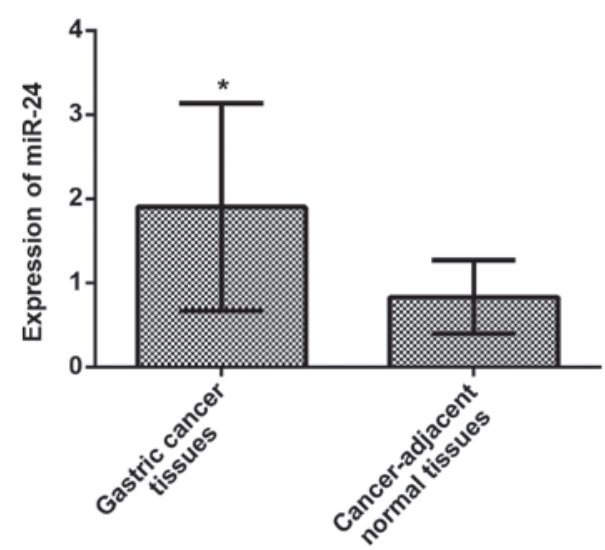

Figure 1. Expression of miR-24 in gastric cancer and cancer-adjacent normal tissues. The expression of miR-24 in gastric cancer and cancer-adjacent normal tissues were detected via RT-qPCR, and the results were statistically analyzed. The expression of miR-24 is $1.906 \pm 1.231$ in gastric cancer tissues and $0.836 \pm 0.437$ in cancer-adjacent normal tissues, and the expression of miR-24 in gastric cancer tissues is significantly higher than that in cancer-adjacent normal tissues $\left(t=10.26,{ }^{*}\right.$ compared with cancer-adjacent normal tissues, $\mathrm{p}<0.01$ ). miR, micro ribonucleic acid; RT-qPCR, reverse transcription-polymerase chain reaction.

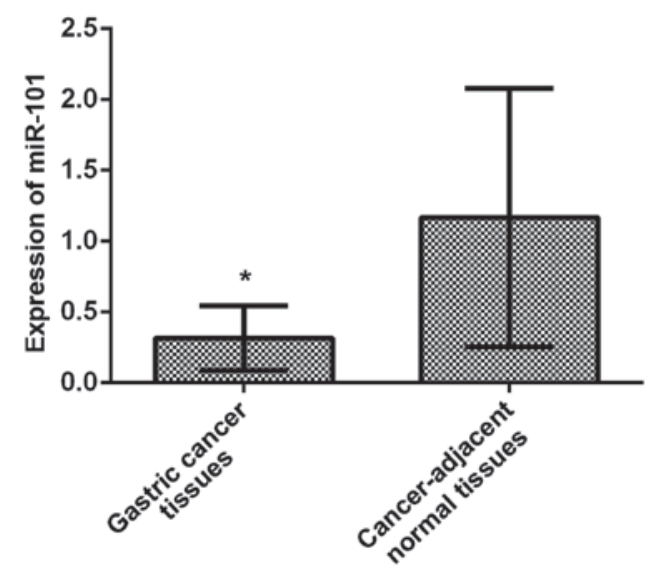

Figure 2. Expression of miR-101 in gastric cancer and cancer-adjacent normal tissues. The expression of miR-101 in gastric cancer and cancer-adjacent normal tissues were detected by RT-qPCR, and the results were statistically analyzed. The expression of miR-101 is $0.316 \pm 0.229$ in gastric cancer tissues and $1.167 \pm 0.914$ in cancer-adjacent normal tissues, and the expression of miR-101 in gastric cancer tissues is significantly lower than that in cancer-adjacent normal tissues $\left(\mathrm{t}=13.940,{ }^{*}\right.$ compared with cancer-adjacent normal tissues, $\mathrm{p}<0.01$ ). miR, micro ribonucleic acid; RT-qPCR, reverse transcription-polymerase chain reaction.

whether miR-24 and miR-101 could be prognostic biomarkers for patients with advanced gastric cancer, $\Delta \Delta \mathrm{Cq}=-2$ was taken

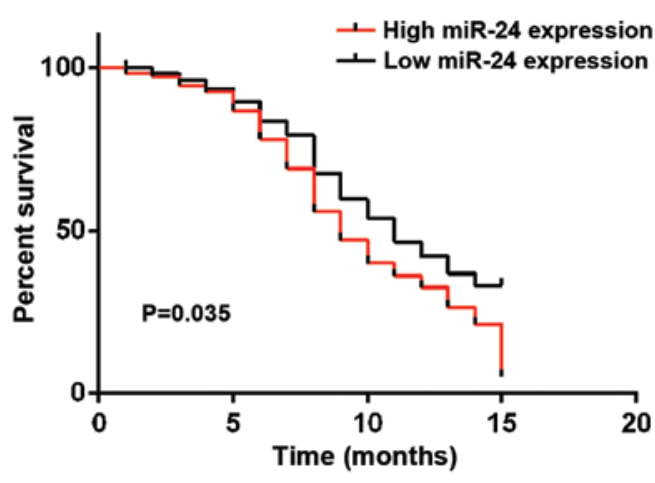

Figure 3. Survival curves of advanced gastric cancer patients with expression of high and low miR-24. The overall survival of patients with advanced gastric cancer is statistically analyzed. The multivariate analysis shows that the overall survival of gastric cancer patients with high miR-24 expression is significantly lower than that of patients with low miR-24 expression $(\mathrm{p}<0.05)$. $\mathrm{miR}$, micro ribonucleic acid.

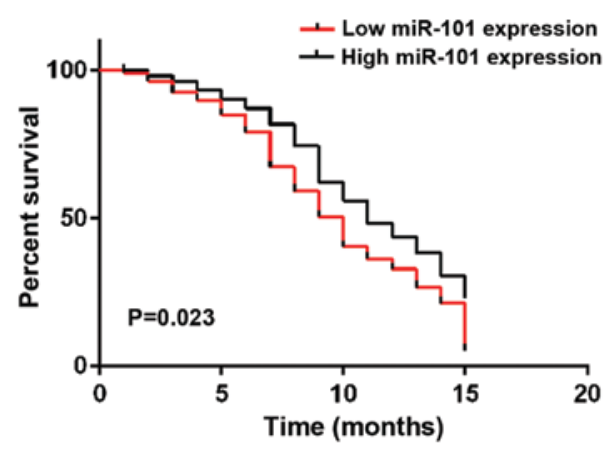

Figure 4. Survival curves of advanced gastric cancer patients with expression of high and low miR-101. The overall survival of patients with advanced gastric cancer was statistically analyzed. The multivariate analysis reveals that the overall survival of gastric cancer patients with low miR-101 expression is significantly lower than that of patients with high miR-101 expression $(\mathrm{p}<0.05)$. miR, micro ribonucleic acid.

as the critical value in this study to determine the expression levels of miR-24 and miR-101. Multivariate analysis showed that the overall survival of patients with high miR-24 expression was shorter than that of patients with low miR-24 expression $(p<0.05)$. The overall survival of patients with low miR-101 expression was shorter than that of patients with high miR-101 expression $(\mathrm{p}<0.05)$ (Figs. 3 and 4$)$.

Correlation of the expression of miR-24 and miR-101 with the survival time of patients with advanced gastric cancer. Cox regression model analyses of general and clinicopathological 
Table II. Correlation of the relative expression levels of miR-24 and miR-101 in gastric cancer tissues with clinicopathological parameters $(\overline{\mathrm{X}} \pm \mathrm{SD})$.

\begin{tabular}{|c|c|c|c|c|c|c|c|}
\hline Types & No. & $\operatorname{miR}-24$ & $\mathrm{~F} / \mathrm{t}$ & P-value & miR-101 & $\mathrm{F} / \mathrm{t}$ & P-value \\
\hline Age & & & 1.074 & 0.284 & & 1.448 & 0.149 \\
\hline$\leq 60$ years & 117 & $1.943 \pm 1.106$ & & & $0.303 \pm 0.351$ & & \\
\hline$>60$ years & 130 & $2.107 \pm 1.276$ & & & $0.376 \pm 0.432$ & & \\
\hline Sex & & & 0.453 & 0.650 & & 0.720 & 0.471 \\
\hline Male & 135 & $1.837 \pm 1.115$ & & & $0.334 \pm 0.386$ & & \\
\hline Female & 112 & $1.769 \pm 1.238$ & & & $0.371 \pm 0.420$ & & \\
\hline Tissue type & & & 1.326 & 0.267 & & 1.389 & 0.251 \\
\hline Adenocarcinoma & 168 & $1.554 \pm 1.371$ & & & $0.341 \pm 0.316$ & & \\
\hline Signet-ring cell carcinoma & 45 & $1.673 \pm 1.535$ & & & $0.298 \pm 0.224$ & & \\
\hline Mucinouss carcinoma & 34 & $1.935 \pm 1.436$ & & & $0.256 \pm 0.228$ & & \\
\hline TNM stage & & & 1.880 & 0.061 & & 1.348 & 0.179 \\
\hline Stage III & 114 & $1.253 \pm 1.011$ & & & $0.410 \pm 0.378$ & & \\
\hline Stage IV & 133 & $1.493 \pm 0.987$ & & & $0.351 \pm 0.310$ & & \\
\hline Tumor differentiation degree & & & 3.950 & 0.020 & & 4.508 & 0.012 \\
\hline High differentiation & 34 & $1.386 \pm 1.296$ & & & $0.376 \pm 0.147$ & & \\
\hline Moderate differentiation & 60 & $1.753 \pm 1.224$ & & & $0.347 \pm 0.153$ & & \\
\hline Low differentiation & 153 & $1.996 \pm 1.436$ & & & $0.264 \pm 0.283$ & & \\
\hline Lymph node metastasis & & & 2.532 & 0.012 & & 2.165 & 0.031 \\
\hline $\mathrm{N} 0+\mathrm{N} 1$ & 131 & $1.176 \pm 0.985$ & & & $0.228 \pm 0.116$ & & \\
\hline $\mathrm{N} 2+\mathrm{N} 3$ & 116 & $1.531 \pm 1.192$ & & & $0.197 \pm 0.108$ & & \\
\hline Tumor site & & & 1.306 & 0.272 & & 1.296 & 0.275 \\
\hline Proximal stomach & 87 & $1.731 \pm 1.537$ & & & $0.397 \pm 0.263$ & & \\
\hline Body of the stomach & 66 & $1.934 \pm 1.883$ & & & $0.354 \pm 0.273$ & & \\
\hline Antrum of the stomach & 94 & $2.163 \pm 1.982$ & & & $0.326 \pm 0.341$ & & \\
\hline Tumor diameter & & & 0.756 & 0.450 & & 1.602 & 0.110 \\
\hline$\leq 5 \mathrm{~cm}$ & 139 & $1.837 \pm 0.972$ & & & $0.374 \pm 0.318$ & & \\
\hline$>5 \mathrm{~cm}$ & 108 & $1.736 \pm 1.124$ & & & $0.309 \pm 0.314$ & & \\
\hline Depth of infiltration & & & 2.493 & 0.013 & & 2.332 & 0.020 \\
\hline $\mathrm{T} 3$ & 37 & $1.413 \pm 1.071$ & & & $0.273 \pm 0.220$ & & \\
\hline $\mathrm{T} 4$ & 210 & $1.893 \pm 1.129$ & & & $0.191 \pm 0.193$ & & \\
\hline
\end{tabular}

miR, micro ribonucleic acid; TNM, tumor-node-metastasis.

Table III. Univariate and multivariate Cox regression analyses of the overall survival of patients with advanced gastric cancer.

\begin{tabular}{|c|c|c|c|c|}
\hline \multirow[b]{2}{*}{ Variables } & \multicolumn{2}{|c|}{ Univariate } & \multicolumn{2}{|c|}{ Multivariate } \\
\hline & HR $(95 \% \mathrm{CI})$ & P-value & $\mathrm{HR}(95 \% \mathrm{CI})$ & P-value \\
\hline Age & $1.969(0.483-3.503)$ & 0.376 & & \\
\hline Sex & $1.890(0.581-6.161)$ & 0.204 & & \\
\hline Pathological differentiation degree & $0.421(0.153-1.177)$ & 0.136 & & \\
\hline Lymph node metastasis & $3.164(1.282-7.333)$ & 0.030 & $2.945(1.344-4.575)$ & 0.211 \\
\hline Depth of infiltration & $2.713(1.523-3.052)$ & 0.026 & $2.391(1.358-3.830)$ & 0.209 \\
\hline miR-24 & $2.945(1.344-4.575)$ & 0.016 & $0.145(0.055-0.391)$ & 0.021 \\
\hline miR-101 & $2.391(1.358-3.830)$ & 0.026 & $3.162(1.324-4.544)$ & 0.019 \\
\hline
\end{tabular}

HR, hazard ratio; CI, confidence interval; miR, micro ribonucleic acid. 
factors were conducted to investigate the effects of the relative expression levels of miR-24 and miR-101 in patients with advanced gastric cancer and clinicopathological features on the overall survival of patients. The results manifested that miR-24, miR-101, lymph node metastasis and the depth of tumor infiltration may be prognostic factors affecting the overall survival of patients with advanced gastric cancer $(p<0.05)$. Meaningful variables confirmed by the univariate analysis were further analyzed via the multivariate Cox regression model. The results revealed that miR-24 and miR-101 were independent predictors affecting the overall survival of patients $(\mathrm{p}<0.01)$ (Table III).

\section{Discussion}

Gastric cancer has become a major disease that threatens the health of human beings all over the world. Its morbidity and mortality rates rank first among malignant tumors (11). The survival time of patients with gastric cancer is often closely related to the clinical stage of the tumor. Many patients did not receive examination due to negligence in the early stage, so that they were diagnosed with advanced gastric cancer. With the increase in the cancer stage, tumor cells gradually infiltrate into the serosal or muscular layer, thus the difficulty of treatment is increased and the overall survival of patients with advanced gastric cancer is not satisfactory $(12,13)$. Clinical treatment for gastric cancer is mainly radical surgery, supplemented by chemotherapy or radiotherapy. Although this method has greatly prolonged the patients' survival time, tumor cell metastasis, invasion and local recurrence are still the main causes of death of patients with the deterioration of advanced gastric cancer (14).

miRNA is a newly discovered non-coding small molecular RNA that affects human physiological and pathological changes. The occurrence and development of gastric cancer are multi-step and multi-factor involved processes, and many miRNAs are abnormally expressed in gastric cancer (15). miR-24 plays a key role in the regulation of cell proliferation, apoptosis, invasion and metastasis in biological characteristics. miR-24 has a dual function: it may play a role as a tumor suppressor gene in various tumor development processes on the one hand, and acts as an oncogene on the other hand (16). miR-24 exerts effects on the occurrence and development of the disease by regulating cell differentiation and cell cycle, and it plays inhibitory roles in BRCA1, CDKN1B and CHEK1 at the $\mathrm{G} 2 / \mathrm{M}$ restriction sites, thus affecting the cell cycle and indirectly inducing the formation and development of the tumor (17). miR-101 is expressed in a variety of eukaryotic organisms, regulates genes, and has multiple targets associated with oncogenes (18). In this study, the expression of miR-24 was significantly upregulated, and the expression of miR-101 was significantly downregulated in gastric cancer tissues compared with those in cancer-adjacent normal tissues, suggesting that miR-24 and miR-101 may be involved in the occurrence and development of gastric cancer and show certain trends in the progressive process of gastric cancer. miR-24 and miR-101 were correlated with the degree of tumor differentiation, lymph node metastasis and depth of infiltration in gastric cancer, indicating that miR-24 and miR-101 can be used as predictive indicators for detecting progression of gastric cancer. In addition, the high expression of miR-24 and the low expression of miR-101 may promote the metastasis and invasion of gastric cancer cells, and the combination of the two may help determine the degree of deterioration of advanced gastric cancer. According to the research of Zhang et al (19) and Chen et al (20), the expression of miR-24 in gastric cancer cell lines and gastric cancer is upregulated in human. miR-24 is related to the process of tumorigenesis of gastric cancer and can induce the occurrence of the tumor by inhibiting the p16 oncogene. The expression of miR-101 is significantly downregulated in gastric cancer tissues, thus losing the inhibitory effect on cyclooxygenase-2 (COX-2) and resulting in the high expression of COX-2 in gastric cancer tissues. Since COX-2 has the ability to promote the generation of tumor-surrounding blood vessels and suppress the apoptosis of tumor cells, the downregulation of miR-101 expression can promote the occurrence and development of gastric cancer. Both miR-24 and miR-101 participate in the metastasis and invasion of gastric cancer. The above is similar to the conclusions of this study.

The overall survival rate of patients with advanced gastric cancer has long been unsatisfactory, and the prognosis of patients has been a hotspot clinically. In this study, the univariate analysis of Cox proportional hazard model for patients with advanced gastric cancer demonstrated that the lymph node metastasis, depth of infiltration, miR-24 and miR-101 may be the prognostic factors affecting the overall survival of patients with advanced gastric cancer. As the impact of confounding factors on the overall survival of patients cannot be controlled in univariate analysis, the intensity of these impacts of indicators observed on the prognosis may be sometimes weakened or enhanced, and false-positive or-negative results may appear; thus, the multivariate Cox model analysis was further performed. The results revealed that the expression of miR-24 and miR-101 was independent prognostic factors for the overall survival of patients, not the lymph node metastasis and depth of infiltration. The study analysis showed that the occurrence of the lymph node metastasis and infiltration in patients represented the further spread of the tumor, and the more serious the disease was, the poorer the prognosis would be, suggesting that miR-24 and miR-101 can be used as indicators for the diagnosis of patients with gastric cancer.

In this study, the selected gastric cancer specimens underwent strict screening to ensure the reliability of the experiment. This study failed to include patients with early gastric cancer, so there were some limitations. The occurrence, development, metastasis and infiltration of the tumor are complex and multi-factor involved processes, and oncogenes may promote the proliferation and apoptosis of tumor cells (21). miR-24 and miR-101 are also abnormally expressed in other tumors, but the mechanisms of the two in gastric cancer remain unclear, so it is expected that the mechanisms of the two in gastric cancer will be elaborated in further studies.

In conclusion, the expression of miR-101 is upregulated and the expression of miR-101 is downregulated in gastric cancer tissues. miR-24 and miR-101 may promote the occurrence, development, infiltration and metastasis of gastric cancer. They may be indicators for the diagnosis of patients with gastric cancer, and have important clinical predictive values. 


\section{Acknowledgements}

Not applicable.

\section{Funding}

No funding was received.

\section{Availability of data and materials}

The datasets used and/or analyzed during the present study are available from the corresponding author on reasonable request.

\section{Authors' contributions}

XD and YL conceived and designed the study, collected, analyzed and interpreted the patient data. XD wrote the manuscript. YL revised the manuscript for important intellectual content. Both authors read and approved the final manuscript.

\section{Ethics approval and consent to participate}

The study was approved by the Ethics Committee of Tianjin Union Medical Centre (Tianjin, China). Signed informed consents were obtained from the patients or guardians.

\section{Patient consent for publication}

Not applicable.

\section{Competing interests}

The authors declare that they have no competing interests.

\section{References}

1. Sitarz R, Skierucha M, Mielko J, Offerhaus GJA, Maciejewski R and Polkowski WP: Gastric cancer: Epidemiology, prevention, classification, and treatment. Cancer Manag Res 10: 239-248, 2018.

2. Torre LA, Siegel RL, Ward EM and Jemal A: Global cancer incidence and mortality rates and trends - an update. Cancer Epidemiol Biomarkers Prev 25: 16-27, 2016.

3. Claassen YHM, de Steur WO, Hartgrink HH, Dikken JL, van Sandick JW, van Grieken NCT, Cats A, Trip AK, Jansen EPM, Kranenbarg WMM, et al: Surgicopathological quality control and protocol adherence to lymphadenectomy in the CRITICS gastric cancer trial. Ann Surg: Aug 16, 2017 (Epub ahead of print).

4. Shimada H, Fukagawa T, Haga Y and Oba K: Does postoperative morbidity worsen the oncological outcome after radical surgery for gastrointestinal cancers? A systematic review of the literature. Ann Gastroenterolog Surg 1: 11-23, 2017.

5. Jang E, Kim E, Son HY, Lim EK, Lee H, Choi Y, Park K, Han S, Suh JS, Huh YM, et al: Nanovesicle-mediated systemic delivery of microRNA-34a for CD44 overexpressing gastric cancer stem cell therapy. Biomaterials 105: 12-24, 2016.

6. Tsai M-M, Wang C-S, Tsai C-Y, Huang H-W, Chi H-C, Lin Y-H, Lu P-H and Lin K-H: Potential diagnostic, prognostic and therapeutic targets of microRNAs in human gastric cancer. Int J Mol Sci 17: 945, 2016.
7. Hyrina A, Olmstead AD, Steven P, Krajden M, Tam E and Jean F: Treatment-induced viral cure of hepatitis $C$ virus-infected patients involves a dynamic interplay among three important molecular players in lipid homeostasis: Circulating microRNA (miR)-24, miR-223, and proprotein convertase subtilisin/kexin type 9. EBioMedicine 23: 68-78, 2017.

8. Pezuk JA, Miller TLA, Bevilacqua JLB, de Barros ACSD, de Andrade FEM, E Macedo LFA, Aguilar V, Claro ANM, Camargo AA, Galante PAF, et al: Measuring plasma levels of three microRNAs can improve the accuracy for identification of malignant breast lesions in women with BI-RADS 4 mammography. Oncotarget 8: 83940-83948, 2017.

9. Sano T, Coit DG, Kim HH, Roviello F, Kassab P, Wittekind C, Yamamoto Y and Ohashi Y: Proposal of a new stage grouping of gastric cancer for TNM classification: International Gastric Cancer Association staging project. Gastric Cancer 20: 217-225, 2017.

10. Livak KJ and Schmittgen TD: Analysis of relative gene expression data using realtime quantitative PCR and the 2(-Delta Delta C(T)) method. Methods 25: 402-408, 2001.

11. Hillard JR: Evaluation of state comprehensive cancer control plans for content related to stomach cancer. J Clin Oncol 34 (4 suppl): 8, 2016.

12. Harvey RC: Second-line treatments for advanced gastric cancer: A network meta-analysis of overall survival using parametric modelling methods. Oncol Ther 5: 53-67, 2017.

13. Kim W, Kim HH, Han SU, Kim MC, Hyung WJ, Ryu SW, Cho GS, Kim CY, Yang HK, Park DJ, et al; Korean Laparoendoscopic Gastrointestinal Surgery Study (KLASS) Group: Decreased morbidity of laparoscopic distal gastrectomy compared with open distal gastrectomy for stage I gastric cancer: Short-term outcomes from a multicenter randomized controlled trial (KLASS-01). Ann Surg 263: 28-35, 2016.

14. Haverkamp L, Ruurda JP, Offerhaus GJ, Weijs TJ, van der Sluis PC and van Hillegersberg R: Laparoscopic gastrectomy in Western European patients with advanced gastric cancer. Eur J Surg Oncol 42: 110-115, 2016.

15. Hsu KW, Fang WL, Huang KH, Huang TT, Lee HC, Hsieh RH, ChiCW and YehTS: Notch1 pathway-mediated microRNA-151-5p promotes gastric cancer progression. Oncotarget 7: 38036-38051, 2016.

16. Lynch SM, McKenna MM, Walsh CP and McKenna DJ: miR-24 regulates $\mathrm{CDKN} 1 \mathrm{~B} / \mathrm{p} 27$ expression in prostate cancer. Prostate 76: 637-648, 2016.

17. Ye SB, Zhang H, Cai TT, Liu YN, Ni JJ, He J, Peng JY, Chen QY, Mo HY, Jun-Cui T, et al: Exosomal miR-24-3p impedes T-cell function by targeting FGF11 and serves as a potential prognostic biomarker for nasopharyngeal carcinoma. J Pathol 240: 329-340, 2016.

18. Chen LG, Xia YJ and Cui Y: Upregulation of miR-101 enhances the cytotoxic effect of anticancer drugs through inhibition of colon cancer cell proliferation. Oncol Rep 38: 100-108, 2017.

19. Zhang H, Duan J, Qu Y, Deng T, Liu R, Zhang L, Bai M, Li J, Ning T, Ge S, et al: Onco-miR-24 regulates cell growth and apoptosis by targeting BCL2L11 in gastric cancer. Protein Cell 7: 141-151, 2016.

20. Chen DL, Ju HQ, Lu YX, Chen LZ, Zeng ZL, Zhang DS, Luo HY, Wang F, Qiu MZ, Wang DS, et al: Long non-coding RNA XIST regulates gastric cancer progression by acting as a molecular sponge of miR-101 to modulate EZH2 expression. J Exp Clin Cancer Res 35: 142, 2016.

21. Soifer HS, Salunga RC, Harris TG, Ramirez J, Gogoi P, Wang Y, Sepehri S, Handique K and Schnabel CA: Gene expression profiling for cancer classification in circulating tumor cells. In: Proceedings of the 107th Annual Meeting of the American Association for Cancer Research. Cancer Res 76 (14 Suppl): Abst 1391,2016

This work is licensed under a Creative Commons Attribution-NonCommercial-NoDerivatives 4.0 International (CC BY-NC-ND 4.0) License. 